\title{
Modified Toluidine Blue: an Alternative Stain for Helicobacter pylori Detection in Routine Diagnostic Use and Post-eradication Confirmation for Gastric Cancer Prevention
}

\author{
Dussadee Sakonlaya ${ }^{1}$, Anucha Apisarnthanarak ${ }^{2}$, Nobutaka Yamada ${ }^{3}$, \\ Prakitpunthu Tomtitchong**
}

\begin{abstract}
Background: Modified toluidine blue staining (MTBs) is a simple, inexpensive and time saving method to detect $\boldsymbol{H}$. pylori in gastric biopsy specimens. As a metachromatic stain, it simultaneously highlights intestinal metaplasia, a gastric cancer precancerous lesion. The aim of this study was to assess the reliability of MTBs compared with hematoxylin-eosin $(\mathrm{H} \& \mathrm{E})$ for $H$. pylori detection using immunoperoxidase staining as the gold standard. This technique would be beneficial for a routine diagnosis and confirmation of $\boldsymbol{H}$. pylori eradication in developing countries where endoscopic-based approaches are dominant. Materials and Methods: Esophagogastroduodenoscopy with triple site gastric biopsies was undertaken in 207 dyspeptic patients at Thammasat University Hospital, Thailand between 1997 and 1999. H\&E, MTBs and immunoperoxidase staining were applied to each specimen. The presence or absence of $\boldsymbol{H}$. pylori with each stain was interpreted separately and the sensitivity, specificity, positive and negative predictive values of $H \& E$ and MTBs were calculated. Results: A total of 282 specimens from 207 patients were evaluated. Using immunoperoxidase staining, organisms were positive in 117 specimens $(41 \%)$. MTBs proved almost equally sensitive as immunoperoxidase (99\%) and significantly more sensitive than H\&E (85\%). It has comparable specificity (96\% vs 96\%), PPV (95\% vs 94\%), and NPV (99\% vs 90\%) to H\&E, using immunoperoxidase staining as gold standard. MTBs compared with immunoperoxidase staining, is cheaper ( 2 USD vs 12 USD) and faster ( $20 \mathrm{~min} v s 16 \mathrm{hrs}$ ) compared to immunoperoxidase staining. Conclusions: MTBs is effective, economical and easy to use in daily practice for the detection of $\boldsymbol{H}$. pylori in gastric biopsy specimens. In addition to saving time in evaluating $\boldsymbol{H}$. pylori associated gastritis, with a high sensitivity and ability to demonstrate intestinal metaplasia, the technique may have a role in confirmation of $\boldsymbol{H}$. pylori eradication for gastric cancer prevention in a developing country setting.
\end{abstract}

Keywords: Helicobacter pylori - histopathology - intestinal metaplasia - modified toluidine blue

Asian Pac J Cancer Prev, 15 (16), 6983-6987

\section{Introduction}

Helicobacter pylori infection is associated with many gastroduodenal diseases such as peptic ulcer disease and gastric cancer (Uemura et al., 2001). The organism is the major cause of chronic active gastritis resulting in chronic atrophic gastritis and its end stage, gastric atrophy, which is believed to be a precancerous lesion (Pandey et al., 2010). Intestinal metaplasia, usually associated with atrophy, is generally regarded as a condition that is predisposed to malignancy as well (Pandey et al., 2010). Several studies have also shown that $H$. pylori played a major role in the pathogenesis of primary gastric lymphoma (Zullo et al., 2014).

Several techniques are available to detect $H$. pylori infection, including biopsy, culture, PCR, imprint cytology, urea breath test, and serology (Barthel and
Everett, 1990; Valentine et al., 1991; Cutler et al., 1995; Matsukura et al., 1995; Thijs et al., 1996; de Boer, 1997). Gastroscopic-based gastric biopsy is one of the most frequently utilized methods. The histologic sections show not only the bacteria but also the morphologic details of the infected mucosa. Although many positive cases can be recognized in a good hematoxylin and eosin (H\&E) stain, a special stain for $H$. pylori should be applied before declaring an inflamed biopsy specimen histologically negative for the organisms (Dixon et al., 1996). The choice of stain, for example, modified giemsa, Warthin-Starry, and Genta stain is a matter of local preference (Genta et al., 1994; Dixon et al., 1996; el-Zimaity et al., 1999). Immunohistochemical methods are highly specific and have an important role in specific situations, but cannot be advocated for the routine diagnosis of $H$. pylori gastritis (Genta et al., 1994). Moreover, to detect concomitant 
intestinal metaplasia, additional staining may be needed (Wright and Kelly, 2006).

Toluidine blue staining in $H$. pylori study was first reported in the literature by Slater in 1990 (Slater, 1990). Our group has optimized the staining technique and has routinely used the modified toluidine blue stain (MTBs) at Nippon Medical School, Japan and Thammasat University Hospital, Thailand since 1996 (Yali et al., 1998; Tomtitchong et al., 1999; Zhang et al., 2001; Zhang et al., 2005a; Zhang et al., 2005b). We found that MTBs is inexpensive; its staining procedure is simple and takes less time when compared to conventional stains such as Warthin-Starry or modified giemsa stain. In addition, with its metachromatic property, the stain can simultaneously demonstrate the acid mucin produced by the intestinal metaplastic cells, if present. Although there are some studies that used this method (Caselli et al., 1997; Dursun et al., 2004; Wright and Kelly, 2006), to our knowledge, there was no study to evaluate the usefulness and the accuracy of this technique. We performed the study to assess the reliability of MTBs compared to $\mathrm{H} \& \mathrm{E}$ stain for $\mathrm{H}$. pylori detection in gastric biopsy specimens using the immunohistochemistry against H. pylori (immunoperoxidase stain, immunostain) as the gold standard. We proposed the use of this technique for routine diagnosis of $H$. pylori and for confirmation of post-therapy bacterial eradication. The latter may have a role in $H$. pylori screening and eradication strategy for gastric cancer prevention in Asian people (Ford et al., 2014), especially in a tropical developing country like Thailand (Wiwanitkit, 2010).

\section{Materials and Methods}

Thammasat University hospital is a tertiary care center in Pathumthani, Central Thailand. In general, dyspeptic patients of our hospital were both seen by gastroenterologists $(60 \%)$ and gastrointestinal surgeons (40\%) depended on the referral system. Ten of surgeons have managed dyspeptic patients in our hospital but in this present study, to avoid misclassification bias during endoscopic classification, all dyspeptic patients presented to one surgical endoscopist (P.T.) during a 2-year period (1997-1999) for esophagogastroduodenoscopy were enrolled and consented to the study. The study was approved by Human Research Ethics Committee of Faculty of Medicine, Thammasat University. Data collected included age, sex, past medical history, past history of dyspepsia and medication, smoking and drinking habits, family history and endoscopic findings. At least three fixed points were biopsied at the antrum, corpus and incisura angularis. Specimens were fixed immediately in $10 \%$ buffered formalin solution prior to the staining process. Paraffin-embedded sections were cut and stained with hematoxylin and eosin (H\&E) and (MTBs). The immunoperoxidase stain against $H$. pylori was applied retrospectively in all unstained sections taken from each set of biopsy specimens. All histologic sections were evaluated by one pathologist (D.S.) who was blinded to the clinical information.

\section{Modified toluidine blue staining (MTBs)}

For preparation of the MTBs, $50 \mathrm{mg}$ of toluidine blue and $10 \mathrm{ml}$ of $0.1 \mathrm{M}$ PBS ( $\mathrm{pH} \mathrm{6.0)} \mathrm{were} \mathrm{mixed} \mathrm{with}$ $90 \mathrm{ml}$ of distilled water. Staining procedure, using the paraffin-embedded tissue, included deparaffinization, washing in distilled water, then incubated with the stain for $15 \mathrm{~min}$, washing well in water, dehydration, clearing, and mounting.

\section{Immunohistochemical study}

Sections were cut and deparaffinized with xylene and $100 \%$ alcohol and treated with a hydrogenperoxidase- $0.3 \%$ methanol solution for $30 \mathrm{~min}$. The sections were pretreated with a protease solution $(0.1 \%$ protease in $0.01 \mathrm{M}$ phosphated-buffered solution (PBS) for $10 \mathrm{~min}$. After cleaning with PBS three times, the sections were incubated with primary antibody against $H$. pylori (polyclonal rabbit anti-Hp, DAKO, California, USA) in a 1:100 solution at $18^{\circ} \mathrm{C}$, overnight. The sections were washed three times with PBS and incubated with 1-2 drops of peroxidaseconjugated swine anti-rabbit for $40 \mathrm{~min}$. After washing with PBS three times, the sections were incubated with 3,3-diaminobenzidine tetrahydrochloride (DAB) at room temperature until a dark brown reaction product was obtained, usually after 5-10 min. Counterstaining was performed with hematoxylin.

\section{Evaluation of the stainings}

The H\&E, MTBs and immunoperoxidase stainings of each case were separately evaluated for the presence or absence of Helicobacter-like organisms at high power magnification (x400). All specimens were examined blindly without knowledge of the identity of the subjects, their clinical data or other stain results.

\section{Evaluation of intestinal metaplasia}

In each case, intestinal metaplasia judged to be positive or negative by presenting of intestinal-type epithelium, including goblet cells, absorptive cells and/or Paneth cells on H\&E and MTBs-stained slides, respectively.

\section{Statistical analysis}

Sensitivity, specificity, positive predictive value (PPV) and negative predictive value (NPV) of MTBs and $H \& E$ stain for detection of $H$. pylori, using the immunoperoxidase stain as the gold standard, were calculated.

\section{Results}

\section{Patient characteristics}

A total of 207 patients were enrolled to the study. Their mean age was $46 \pm 17.5$ years (range: $16-85)$. This involved 116 males (56\%) and 91 females (44\%). Peptic ulcer disease was diagnosed in 81 of the 207 patients $(39.1 \%)$. There were 43 patients $(20.8 \%)$ with duodenal ulcer, 30 patients $(14.5 \%)$ with gastric ulcer and 8 patients $(3.9 \%)$ with gastroduodenal ulcers. Gastritis was diagnosed in 42 patients $(20.3 \%)$. In another 61 patients $(29.5 \%)$, the diagnosis was non-ulcer dyspepsia. The remaining patients were diagnosed as duodenitis $(n=6,2.9 \%)$, erosive 
gastroduodenitis $(n=1,0.5 \%)$, gastric erosion $(n=6,2.9 \%)$, hypertensive gastropathy $(n=1,0.5 \%)$, gastroesophageal reflux disease $(n=5,2.4 \%)$ and gastric carcinoma $(n=4$, $1.9 \%$ ). The total number of endoscopic biopsies in these 207 patients was 282 .

\section{The stains}

Although visible, $H$. pylori are relatively difficult to detect with H\&E stain. In contrast, they are easily detected in the MTBs and the immunoperoxidase-stained sections. For economical evaluation, the immunostaining process takes 16 hours and costs 12 USD for each staining. Meanwhile, the whole MTBs staining process will take 20 minutes and the cost of MTBs is 2 USD each.

By MTBs staining, the organisms are dark blue against a variable blue background. They are typically $0.5 \mu \mathrm{m}$ wide, 1-3 $\mu \mathrm{m}$ long, curved spiral-rod in shape confined to the mucus layer, closely adherent to the mucosal surface, and/or within the gastric pits (Figure 1). In addition, the MTBs can simultaneously demonstrate neutrophilic infiltration in the glands and stroma. The nuclear lobes of the neutrophils stand out against their clear unstained cytoplasm.

H. pylori were identified on immunoperoxidasestained slides in 117 of 282 biopsy specimens (41\%). The organisms stain brown and are located at the same sites as in MTBs-stained sections. In addition, they are found in the cytoplasm of neutrophils, focally.

Intestinal metaplasia was identified by $H \& E$ and MTBs in 37 specimens (13\%). On MTBs-stained slides, the metaplastic cells stain magenta contrasting with the blue background (Figure 2). Although no additional foci of intestinal metaplasia were seen by MTBs stain, the detection of small areas of incomplete metaplasia with scattered goblet cells was much easier.

\section{Diagnostic test results}

The diagnostic accuracy of each stain was shown in

Table 1. Sensitivity, Specificity, Positive and Negative Predictive value of $\mathrm{H} \& \mathrm{E}$ and MTBs for Detection of H. pylori Compared with Immunoperoxidase Stain, as the Gold Standard

\begin{tabular}{lcccc}
\hline Staining methods & Sensitivity (\%) & Specificity (\%) & PPV (\%) & NPV (\%) \\
\hline H\&E & 85 & 96 & 94 & 90 \\
MTBs & 99 & 96 & 95 & 99 \\
\hline
\end{tabular}

*H\&E=hematoxylin and eosin stain; MTBs=modified toluidine blue stain; PPV=positive predictive value; NPV=negative predictive value

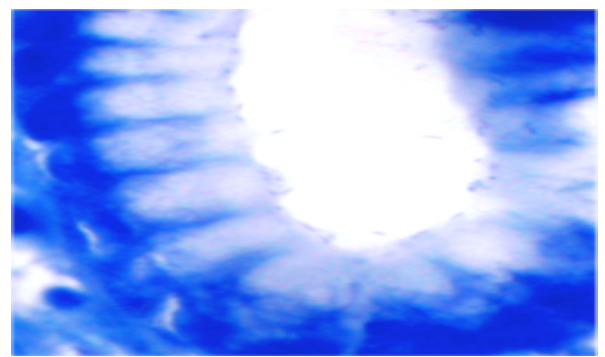

Figure 1.H.pylori are typically Curved Spiral-Rod in Shape, Confined to the Mucus Layer, Closely Adherent to the Mucosal Surface and within the Gastric Pits. (MTBs, original magnification $\mathrm{x} 400$ )

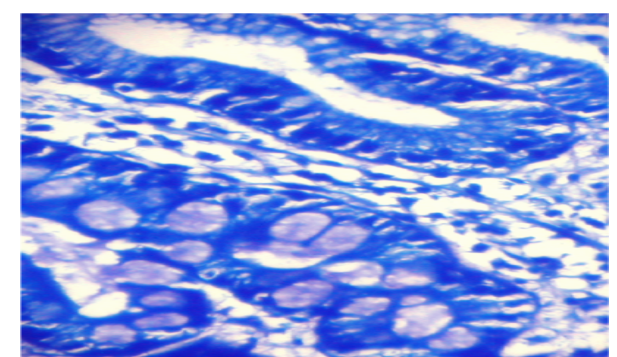

Figure 2. The Metaplastic Cells Stain Magenta, in Contrast to the Blue Background Indicated Intestinal Metaplasia. (MTBs, original magnification x400)

Table 1. MTBs was almost equally sensitive in detecting H. pylori as the immunostain. H\&E was less sensitive (85\%) than MTB (99\%) using immunostain as gold standard. MTBs also had higher negative predictive value (99\%) in determining H. pylori infection than H\&E (90\%). The specificity and positive predictive value of $H \& E$ were $96 \%$ and $94 \%$; MTBs, $96 \%$ and $95 \%$, respectively.

Specimens, which were interpreted differently on different stains, were carefully reviewed to determine the nature of the discrepancies. There were 8 positive $\mathrm{H} \& \mathrm{E}$ stains with negative immunoperoxidase stain. Six of these cases were positive for MTBs staining. On second evaluation, two cases with negative MTBs stains were considered to be false positive $H \& E$ stains due to mucus debris that was erroneously interpreted as H.pylori.

\section{Discussion}

It should be noted that the importance of detecting $H$. pylori has crossed over from the research laboratory to clinical practice. The need to eradicate $H$. pylori as part of the management of peptic ulcer disease and gastric cancer prevention is increasingly recognized by clinicians. Meanwhile a new and effective treatment regimen for $H$. pylori is becoming available, confirmation of eradication is crucial and is best obtained by the histopathologic examination of post-treatment biopsy specimens (Genta et al., 1994).

Toluidine blue staining in H.pylori study was firstly reported in the literature from 1990. Our group from Japan and Thailand had optimized the staining technique and used it routinely since 1996 . We found that it was a very easy, fast and cheap for $H$. pylori identification comparing with other standard techniques. We firstly reported the use of it in the literature from the year 1998 (Yali et al., 1998). Nevertheless, one may question the usefulness and the accuracy of this technique.

In a clinical setting of tropical developing country in Thailand where availability of Urea Breath Test (UBT) is still very limited and endoscopic-based diagnosis is widely used, we present our data of MTBs in comparison with immunostain as standard. This confirms that our technique could be used as an alternative technique for definitive diagnosis of $H$. pylori with a very high economic benefit. The sensitivity of the MTBs in detecting $H$.pylori is almost similar to that of the immunostain, which is currently used as a gold standard in a recent publication (Yu et al., 2014). Moreover, its negative predictive value 
of $99 \%$ indicates that it is the reliable method to exclude H. pylori infection, if negative. The limitation of this study is the sensitivity and specificity of MTBs may vary among different observers depending on the experience of pathologists in identifying the organisms.

MTBs enhances the visualization of bacteria, particularly in a specimen with low density of H.pylori, including the post-eradication cases, in small-sized biopsies containing few glands, and in case of abundant mucus debris on the surface or pits. The stain also highlights the neutrophilic infiltration which is highly associated with $H$. pylori infection. Furthermore, the cost of the reagents is much lower than that of Warthin-Starry and immunoperoxidase stains. The staining process is also simpler and the time needed for testing is reduced (within 20 minutes). We propose the use of MTBs technique as a cost-effectiveness tool for $H$. pylori diagnosis and underlying the use in a follow-up period after bacterial eradication in gastric cancer prevention strategy for Asian people (Ford et al., 2014), especially for a clinical setting in developing country (Wiwanitkit, 2010).

There are two possible explanations for the discrepancies between MTBs and immunoperoxidase stain: the patchy distribution of $H$. pylori in gastric samples and/or focal loss of the organisms during the staining process. Therefore, to avoid such false negative results, adequate specimens are necessary. For adequacy, specimens from antrum together with corpus and incisura angularis are recommended. Corpus biopsies are particularly valuable for yielding positive results after treatment, especially where proton pump inhibitors have been used. Under these circumstances, organisms may become rare or disappear from the antrum but remain in the oxyntic mucosa. However, maximal degrees of atrophy and intestinal metaplasia are consistently found in the region of the incisura angularis, which is also the site most likely to reveal premalignant dysplasia (Dixon et al., 1996).

Many laboratories routinely employ an Alcian blue ( $\mathrm{pH} 2.5$ )/periodic acid Schiff stain on gastric specimens to demonstrate intestinal metaplasia (Dixon et al., 1996). Acid mucin in metaplastic cells stains blue or purple of the Alcian blue, in contrast to the Schiff-positive neutral mucin presented in the surface and foveolar epithelium and the mucus glands of the non-metaplastic gastric mucosa. In light of the knowledge that acid mucin can be demonstrated by the metachromatic stains (Cook, 1996), MTBs with its metachromatic property, can be applied for effective demonstration of intestinal metaplasia. Using the stain allows simultaneous evaluation of $H$. pylori infection status, gastritis activity and intestinal metaplasia on a single slide. Although MTBs does not enhance the sensitivity for detection of intestinal metaplasia, it is easily recognizable and provides a more precise assessment of the extension.

In conclusion, MTBs is effective, economical and easy to use in daily practice for detection of $H$. pylori on gastric biopsy specimens in addition to routine histologic sections. With its high sensitivity to the organisms and the ability to demonstrate intestinal metaplasia and to highlight the neutrophilic infiltration, it lessens the time necessary to evaluate $H$. pylori-associated gastritis. MTBs may be beneficial for confirmation of bacterial eradication for gastric cancer prevention in developing country.

\section{Acknowledgements}

The authors would like to thank Dr. Pachongjit Suwanprakorn, from Faculty of Medicine, Thammasat University for encouragement and financial support. The authors are also indebted to Prof. Masaru Miki from Nippon Medical School, Tokyo for his valuable support for our training in Japan. The authors are indebted to Prof. David Y. Graham from Michael E. DeBakey Veterans Affairs Medical Center, Houston, Texas, USA for his valuable comment. This work was partially supported by the National Research University Project of Thailand Office of Higher Education Commission.

Part of this work was presented at XXV Congress of the International Academy of Pathology 2004 in Brisbane, Queensland, Australia and the abstract of this work is published in Pathology International, 2004, Vol. 54 (Supple.2), page A82-A83. The reason for the delay in publishing the full text is that difficulties were encountered in compiling the collaborative work of the various investigators, due to the retirement and/or relocation of some of them.

\section{References}

Barthel JS, Everett ED (1990). Diagnosis of Campylobacter pylori infections: the "gold standard" and the alternatives. Rev Infect Dis, 12, 107-14.

Caselli M, Trevisani L, Tursi A, et al (1997). Short-term lowdose triple therapy with azithromycin, metronidazole and lansoprazole appears highly effective for the eradication of Helicobacter pylori . Eur J Gastroenterol Hepatol, 9, 45-8.

Cook HC (1996). Carbohydrate. In "Theory and Practice of Histological Techniques", Eds Bancroft JD and Stevens A. Churchill Livingstone, New York pp 173-211.

Cutler AF, Havstad S, Ma CK, et al (1995). Accuracy of invasive and noninvasive tests to diagnose Helicobacter pylori infection. Gastroenterology, 109, 136-41.

de Boer WA (1997). Diagnosis of Helicobacter pylori infection. Review of diagnostic techniques and recommendations for their use in different clinical settings. Scand J Gastroenterol, 223, 35-42.

Dixon MF, Genta RM, Yardley JH, et al (1996). Classification and grading of gastritis. The updated Sydney System. International Workshop on the Histopathology of Gastritis, Houston 1994. Am J Surg Pathol, 20, 1161-81.

Dursun M, Yilmaz S, Yukselen V, et al (2004). Evaluation of optimal gastric mucosal biopsy site and number for identification of Helicobacter pylori, gastric atrophy and intestinal metaplasia. Hepatogastroenterology, 51, 1732-5.

el-Zimaity HM, Wu J, Graham DY (1999). Modified Genta triple stain for identifying Helicobacter pylori. J Clin Pathol, 52, 693-4.

Ford AC, Forman D, Hunt RH, et al (2014). Helicobacter pylori eradication therapy to prevent gastric cancer in healthy asymptomatic infected individuals: systematic review and meta-analysis of randomised controlled trials. BMJ, 348, 3174.

Genta RM, Robason GO, Graham DY (1994). Simultaneous visualization of Helicobacter pylori and gastric morphology: 
a new stain. Hum Pathol, 25, 221-6.

Matsukura N, Onda M, Tokunaga A, et al (1995). Detection of Helicobacter pylori DNA in gastric juice by the polymerase chain reaction: comparison with findings in bacterial culture and the detection of tissue $\operatorname{IgA}$ and serum IgG antibodies against Helicobacter pylori. J Gastroenterol, 30, 689-95.

Pandey R, Misra V, Misra SP, et al (2010). Helicobacter pylori and gastric cancer. Asian Pac J Cancer Prev, 11, 583-8.

Slater B (1990). Superior stain for Helicobacter pylori using toluidine O. J Clin Pathol, 43, 961.

Thijs JC, van Zwet AA, Thijs WJ, et al (1996). Diagnostic tests for Helicobacter pylori: a prospective evaluation of their accuracy, without selecting a single test as the gold standard. Am J Gastroenterol, 91, 2125-9.

Tomtitchong P, Tiemtanom S, Eiumtrakul S, et al (1999). $H$. pylori infection and the correlation to chronic active gastritis detected by the histological division of the Sydney System. Siriraj Hosp Gaz, 51, 339-47.

Uemura N, Okamoto S, Yamamoto S, et al (2001). Helicobacter pylori infection and the development of gastric cancer. $N$ Engl J Med, 345, 784-9.

Valentine JL, Arthur RR, Mobley HL, et al (1991). Detection of Helicobacter pylori by using the polymerase chain reaction. J Clin Microbiol, 29, 689-95.

Wiwanitkit V (2010). Helicobacter pylori screening to prevent gastric cancer: an economical analysis for a tropical developing country. Asian Pac J Cancer Prev, 11, 571-2.

Wright CL, Kelly JK (2006). The use of routine special stains for upper gastrointestinal biopsies. Am J Surg Pathol, 30, 357-61.

Yali Z, Yamada N, Wen M, et al (1998). Gastrospirillum hominis and Helicobacter pylori infection in Thai individuals: comparison of histopathological changes of gastric mucosa. Pathol Int, 48, 507-11.

Yu XW, Xu Q, Xu Y, et al (2014). Expression of the E-cadherin/ beta-catenin/tcf-4 pathway in gastric diseases with relation to Helicobacter pylori infection: clinical and pathological implications. Asian Pac J Cancer Prev, 15, 215-20.

Zhang C, Yamada N, Wu YL, et al (2005a). Comparison of Helicobacter pylori infection and gastric mucosal histological features of gastric ulcer patients with chronic gastritis patients. World J Gastroenterol, 11, 976-81.

Zhang C, Yamada N, Wu YL, et al (2005b). Helicobacter pylori infection, glandular atrophy and intestinal metaplasia in superficial gastritis, gastric erosion, erosive gastritis, gastric ulcer and early gastric cancer. World J Gastroenterol, 11, 791-6.

Zhang Y, Lai Z, Wang J (2001). Helicobacter pylori associated gastric mucosal inflammation and histopathological assessment. Zhonghua Yi Xue Za Zhi, 81, 811-5 (in Chinese).

Zullo A, Hassan C, Ridola L, et al (2014). Gastric MALT lymphoma: old and new insights. Ann Gastroenterol, 27, 27-33. 